MIRANDA, N.O.; OLIVEIRA, T.S.; MEDEIROS, J.F.; SILVA, C.A. Variabilidade espacial da produtividade do meloeiro em áreas de cultivo fertirrigado. Horticultura Brasileira, Brasília, v.23, n.2, p.260-265, abr-jun 2005.

\title{
Variabilidade espacial da produtividade do meloeiro em áreas de cultivo fertirrigado ${ }^{1}$
}

\author{
Neyton de O. Miranda ${ }^{2}$; Teógenes S. de Oliveira ${ }^{3}$; José Francismar de Medeiros ${ }^{2}$; Cleusirene A. da Silva ${ }^{2}$ \\ ${ }^{2}$ ESAM, C. Postal 137, 59.625-900 Mossoró-RN; E-mail: neyton@esam.br ${ }^{3}$ UFC, Depto. Ciências do Solo.
}

\begin{abstract}
RESUMO
Duas áreas de produção de melão fertirrigado por gotejamento foram amostradas em malha com objetivo de identificar a variabilidade espacial de variáveis de produção, determinar sua dependência espacial e localizar regiões que, manejadas localizadamente, obtenham melhor retorno econômico. Na primeira área, em solo classificado como Latossolo Vermelho Amarelo eutrófico, utilizou-se o híbrido Goldex, e na outra, em Argissolo Vermelho Amarelo, o híbrido 'Orange Flesh'. Foram determinados o estande, a produção de frutos dos tipos exportação, mercado nacional e comerciável. Utilizou-se a estatística descritiva para levantamento exploratório dos dados e a geoestatística para avaliar a dependência espacial das variáveis e confeccionar mapas de isovalores por interpolação. As variáveis, com exceção do estande, se ajustaram à distribuição normal e apresentaram variabilidade média, com dependência espacial e ajuste aos modelos de semivariograma exponencial ou esférico. Utilizando-se mapas de isovalores, identificaram-se regiões onde o manejo localizado pode aumentar o retorno econômico caso identificados os fatores limitantes e novas tecnologias sejam usadas adequadamente.
\end{abstract}

Palavras-chave: Cucumis melo, geoestatística, manejo localizado.

\begin{abstract}
Spatial variability of melon yield under fertigation in Mossoró, Brazil

Two production areas of trickle fertigated melon were grid sampled with the objective of assessing spatial variability of yield, and to determine the spatial dependence while locating regions where site specific management can improve economic returns. The hybrid Goldex was grown in a Yellow Red Latosol, and the Orange Flesh in a Yellow Red Argisol. The stand, yield of export type fruits, yield of national market fruits and marketable yield were determined. Descriptive statistics was used for an exploratory analysis of data, while geostatistics was used for evaluating spatial dependence of variables and generate contour maps by interpolation techniques. All variables, except stand, adjusted to normal distribution and showed medium variability. Yield variables were spatially dependents and adjusted to exponential or spherical models of semi-variograms. Contour maps showed regions where site specific management can improve economic returns since limiting factors are identified and suitable technologies adopted.
\end{abstract}

Keywords: Cucumis melo, geostatistics, site specific management.

\section{(Recebido para publicação em 25 de março de 2004 e aceito em 7 de abril de 2005)}

$\mathrm{O}$ melão é um importante produto do agronegócio brasileiro, sendo os estados do RN, CE, BA e PE responsáveis por $99 \%$ da produção. As exportações de melão geraram para o país uma renda de 221,4 milhões de dólares no ano de 2001, enquanto que no ano de 2002, somente pelo porto de Natal, foram exportados $98 \mathrm{mil}$ toneladas de frutos de melão que geraram uma renda aproximada de 39 milhões de dólares (CRISÓSTOMO et al., 2002; SILVA; COSTA, 2003).

A cultura do melão recebe um manejo convencional, no qual as práticas culturais são aplicadas uniformemente porque se assume a homogeneidade dos fatores de produção em todo o campo. Porém, segundo Cassel et al., (1988) e Marques Júnior e Corá (1998), o desconhecimento pontual dos fatores de produção torna metas de produtividade impróprias e leva ao uso excessivo de insumos, tornando a produção ineficiente com altos custos e danos ambientais.

A variabilidade de um campo agrícola influencia fatores de produção ligados à disponibilidade de nutrientes, suprimento de água e, conseqüentemente, ao ambiente onde estão localizadas as raízes (MANTOVANI et al., 1998). Se for constatada alta variabilidade espacial dos fatores produtivos e da produtividade das culturas, inclusive do melão, a localização das regiões de alto e baixo potencial produtivo pode trazer benefícios pela adoção de estratégias como a aplicação localizada de insumos e tratos culturais (MORTON et al., 1998; NIELSEN et al., 1997; PLANT et al., 1999).

A variabilidade na produtividade de uma cultura dentro do campo pode ser inerente do solo, clima ou induzida pelo manejo. Entre modificações nas propriedades físicas, químicas e biológicas do solo causadas por práticas de manejo, estão a compactação do solo pelas máquinas agrícolas e a variabilidade no estande das culturas e nos teores de nutrientes no solo devido à aplicação desuniforme de sementes, fertilizantes e corretivos (CASSEL et al., 1988; PLANT et al., 1999; RUNGE e HONS, 1998).

O primeiro passo para produzir melão considerando-se a variabilidade espacial é a confecção de mapas de produtividade, os quais, segundo Broos et al., (1998) e Staford et al. (1998), permitem visualizar a variabilidade dos fatores de produção e da produtividade das culturas. A análise destes mapas e a correlação entre propriedades do solo e da cultura possibilitam decisões de mane-

\footnotetext{
${ }^{1}$ Parte da tese apresentada à UFC para obtenção do grau de doutor em Agronomia-Fitotecnia.
} 
jo sobre irrigação e drenagem, plantio, variedades adaptadas, aplicação localizada de sementes, fertilizantes e corretivos, herbicidas e defensivos agrícolas, e intensidade do preparo do solo (SEARCY, 2000; SCHUELLER; WHITNEY, 2000). Mapas de isovalores são gerados por interpolação a partir das quantidades de produto amostradas em um conjunto de muitos pontos, ou células com área e posição geográfica conhecida (MANTOVANI et al., 1998).

O presente trabalho teve como objetivo avaliar a variabilidade espacial de variáveis de produção do meloeiro em duas áreas de produção comercial com diferentes solos, híbridos e tratos culturais, de forma a determinar a dependência espacial vinculada a fatores que, sendo identificados, permitam indicações para o manejo racional da cultura.

\section{MATERIAL E MÉTODOS}

O trabalho foi realizado na Fazenda São João, Mossoró, com coordenadas geográficas $05^{\circ} 09^{\prime} 59,55^{\prime}$ ' S e $37^{\circ} 24^{\prime}$ 39,92" W, altitude de $29 \mathrm{~m}$, de 16/08/ 2002 a 04/01/2003, com os híbridos de melão Goldex e Orange Flesh. O híbrido Goldex foi cultivado numa área de 3,18 ha em solo classificado como Latossolo Vermelho Amarelo eutrófico, e o Orange Flesh em uma área de 1,92 ha em Argissolo Vermelho Amarelo.

O preparo do solo em ambas as áreas constou de duas gradagens para fragmentação e incorporação da vegetação ao solo, subsolagem, nova gradagem e sulcamento para aplicação de $4 \mathrm{tha}^{-1} \mathrm{de}$ composto $\left(1,3 \%\right.$ de $\mathrm{N} ; 0,91 \%$ de $\mathrm{P}_{2} \mathrm{O}_{5}$ $3 \%$ de $\mathrm{K}_{2} \mathrm{O} ; 3,6 \%$ de $\mathrm{Ca}$ e $0,5 \%$ de $\mathrm{Mg}$ ). A semeadura foi feita em bandejas de 128 células e o transplante aconteceu após 12 dias. No 'Goldex', cuja população foi de 16.667 plantas ha ${ }^{-1}$, o espaçamento foi de $2,0 \times 0,5 \mathrm{~m}$, com três plantas por gotejador, sendo que a cada três gotejadores um recebia apenas duas plantas. No 'Orange Flesh', cuja população foi de 18.750 plantas ha-1, o espaçamento foi de $2,0 \times 0,8 \mathrm{~m}$, com três plantas por gotejador. Os demais tratos culturais seguiram as recomendações para a cultura.

Os tubos gotejadores apresentavam emissores não autocompensantes, do tipo labirinto, espaçados de 0,5 m, com vazão de 2,27 $\mathrm{L} \mathrm{h}^{-1}$ no 'Goldex', e autocompensantes, espaçados de $0,8 \mathrm{~m}$, com vazão de 5,0 $\mathrm{L} \mathrm{h}^{-1}$ no 'Orange Flesh'. A pressão de operação foi de $245 \mathrm{kPa}$ no cabeçal de controle. O manejo da irrigação se baseou na evapotranspiração de referência calculada pelo método de Penman Monteith com dados da Estação Climatológica da ESAM, com coeficientes de cultura propostos pela FAO (ALLEN et al., 1998), e em características do solo. As lâminas totais de irrigação, entre 11 e 79 dias após semeadura, foram de $322,8 \mathrm{~mm}$ no Latossolo e de 316,7 mm no Argissolo. A adubação via água de irrigação, baseada em análises do solo e recomendações para a cultura, constou de $70 \mathrm{~kg} \mathrm{ha}^{-1}$ de N, $138 \mathrm{~kg}$ ha $^{-1}$ de $\mathrm{P}_{2} \mathrm{O}_{5}, 267 \mathrm{~kg} \mathrm{ha}^{-1}$ de $\mathrm{K}_{2} \mathrm{O}, 2,2 \mathrm{~kg}$ $\mathrm{ha}^{-1}$ de $\mathrm{B}, 3,81 \mathrm{~kg} \mathrm{ha}^{-1}$ de $\mathrm{CaO}, 1,4 \mathrm{~kg}$ $\mathrm{ha}^{-1} \mathrm{de} \mathrm{MgO}$ e 9,4 $\mathrm{kg} \mathrm{ha}^{-1}$ de $\mathrm{S}$, no Latossolo; e $90 \mathrm{~kg} \mathrm{ha}^{-1}$ de N, $121 \mathrm{~kg} \mathrm{ha}^{-1}$ de $\mathrm{P}_{2} \mathrm{O}_{5}, 318 \mathrm{~kg} \mathrm{ha}^{-1}$ de $\mathrm{K}_{2} \mathrm{O}, 6,5 \mathrm{~kg}$ ha ${ }^{1} \mathrm{de} \mathrm{B}, 2,45 \mathrm{~kg}$ de $\mathrm{CaO}, 7 \mathrm{~kg} \mathrm{ha}^{-1} \mathrm{de}$ $\mathrm{MgO}, 4,8 \mathrm{~kg} \mathrm{ha}^{-1}$ de $\mathrm{S}$ e 0,2 kg de Zn, no Argissolo.

As amostragens foram realizadas em 75 parcelas de $20 \mathrm{~m}^{2}$ no Latossolo e 56 parcelas de 11,2 $\mathrm{m}^{2}$ no Argissolo. Estas parcelas estavam centralizadas nos nós de uma malha locada em espaçamento de $20 \mathrm{~m}$ no Latossolo e de $19 \mathrm{~m}$ no Argissolo. Foi determinado o número de plantas por parcela (estande) e realizadas duas colheitas no 'Goldex' e quatro no 'Orange Flesh'. Os frutos dos dois híbridos foram classificados de forma semelhante nos tipos exportação (PEXP), mercado nacional (PNAC) e comerciável (PEXP+PNAC), e expressos em t ha-1. Os do tipo exportação, segundo Moretti e Araújo (2003), eram os frutos menores, com massa ao redor de $1,0 \mathrm{~kg}$ e de forma arredondada. Em geral são frutos do tipo 10 e acima, ou seja, uma caixa padrão contém dez ou mais frutos, até quatorze. Além disso, é exigida alta qualidade das características do produto, como consistência, uniformidade de formato, tamanho, cor e teor de açúcares. Os frutos tipo mercado nacional são aqueles comercializados no mercado local, regional ou nacional. Os refugados são aqueles com evidentes defeitos de forma, tamanho, manchas, danos mecânicos, cor e sanidade.
Utilizou-se a estatística descritiva para analisar o comportamento geral dos dados e a aderência à distribuição normal e procedimentos de geoestatística, através dos softwares GeoEAS 1.2.2 (ENGLUND; SPARKS, 1991) e Variowin (PANNATIER, 1996), para confeccionar semivariogramas "omnidirecionais" e determinar o modelo de melhor ajuste, bem como avaliar a dependência espacial e produzir mapas de isovalores por krigagem.

\section{RESULTADOS E DISCUSSÃO}

Observou-se pequena diferença entre médias e medianas das variáveis PEXP, PNAC, PCOM e estande dos híbridos Goldex e Orange Flesh, indicando distribuições simétricas e normalidade dos dados que, com exceção do estande, foi confirmada pelo teste de Shapiro-Wilk. Segundo Johnson et al. (1998) uma grande amplitude, como a que foi encontrada para os dados de produtividade, indica a existência de variabilidade de características do solo da qual se pode tirar proveito através do manejo localizado.

As produtividades médias de frutos no 'Goldex' e no 'Orange Flesh' foram, respectivamente, de 11,36 $\mathrm{t} \mathrm{ha}^{-1}$ e 12,18 $\mathrm{t} \mathrm{ha}^{-1}$ no tipo exportação (PEXP); 13,68 $\mathrm{t} \mathrm{ha}^{-1}$ e $6,23 \mathrm{t} \mathrm{ha}^{-1}$ no tipo mercado nacional (PNAC) e 25,18 $\mathrm{t} \mathrm{ha}^{-1}$ e $18,50 \mathrm{t}$ ha $^{-1}$ no tipo comerciável (PCOM). O estande foi de 32,33 plantas por parcela no 'Goldex' e de 20,57 no 'Orange Flesh', quando se esperavam 33 e 21 plantas, respectivamente. Apesar da desuniformidade na população de plantas poder influenciar a produtividade, qualidade e retorno econômico (STOMBAUGH; SHEARER, 2000), a baixa variabilidade do estande (CV de $6,21 \%$ e $3,92 \%$, respectivamente para 'Goldex' e 'Orange Flesh') indica não ter havido este tipo de problema. Os CVs das outras variáveis indicam variabilidade média de acordo com critério adotado por Warrick (1998), sendo os maiores referentes a PNAC $(34,62 \%$ para 'Goldex' e 48,36\% para 'Orange Flesh') e PEXP (40,55\% para 'Goldex' e 28,57\% para 'Orange Flesh') e os menores de PCOM (21,87\% para 'Goldex' e 28,90\% para 'Orange Flesh'). 
Tabela 1. Parâmetros dos modelos ajustados aos semivariogramas das variáveis de produção de melão 'Goldex' e 'Orange Flesh'. Mossoró, ESAM, 2002.

\begin{tabular}{|c|c|c|c|c|c|c|c|c|}
\hline \multirow{2}{*}{$\begin{array}{l}\text { Parâmetros } \\
\text { Híbrido }\end{array}$} & \multicolumn{2}{|c|}{$\operatorname{PEXP}^{1}\left(\mathrm{t} \mathrm{ha}^{-1}\right)$} & \multicolumn{2}{|c|}{$\operatorname{PNAC}^{2}\left(\mathrm{t} \mathrm{ha}^{-1}\right)$} & \multicolumn{2}{|c|}{$\operatorname{PCOM}^{3}\left(\mathrm{t} \mathrm{ha}^{-1}\right)$} & \multicolumn{2}{|c|}{ ESTANDE $\left(\mathrm{n}^{\circ}\right)$} \\
\hline & G & OF & $G$ & OF & $G$ & OF & $G$ & OF \\
\hline Modelo & EXP $^{4}$ & $\mathrm{ESF}^{5}$ & EXP & EXP & ESF & EXP & $\mathrm{EPP}^{6}$ & EPP \\
\hline$\overline{\mathrm{C}_{0}{ }^{7}}$ & 3,00 & 0,00 & 7,02 & 3,00 & 0,00 & 8,80 & 3,80 & 0,665 \\
\hline$\left(C_{0}+C 1\right)^{8}$ & 18,22 & 13,18 & 13,60 & 6,12 & 29,60 & 20,00 & - & - \\
\hline$a(m)^{9}$ & 35,50 & 41,25 & 30,30 & 67,30 & 29,43 & 58,30 & - & - \\
\hline $\mathrm{C}_{0} /\left(\mathrm{C}_{0}+\mathrm{C}_{1}\right)^{10}$ & $16 \%$ & $<1 \%$ & $52 \%$ & $49 \%$ & $<1 \%$ & $44 \%$ & - & - \\
\hline
\end{tabular}

${ }^{1}$ Produção de frutos tipo exportação; ${ }^{2}$ Produção de frutos tipo mercado nacional; ${ }^{3}$ Produção de frutos comerciáveis; ${ }^{4}$ Exponencial; ${ }^{5}$ Esférico; ${ }^{6}$ Efeito pepita puro; ${ }^{7}$ Efeito Pepita; ${ }^{8}$ Patamar; ${ }^{9}$ Alcance; ${ }^{10}$ Relação efeito pepita/patamar.

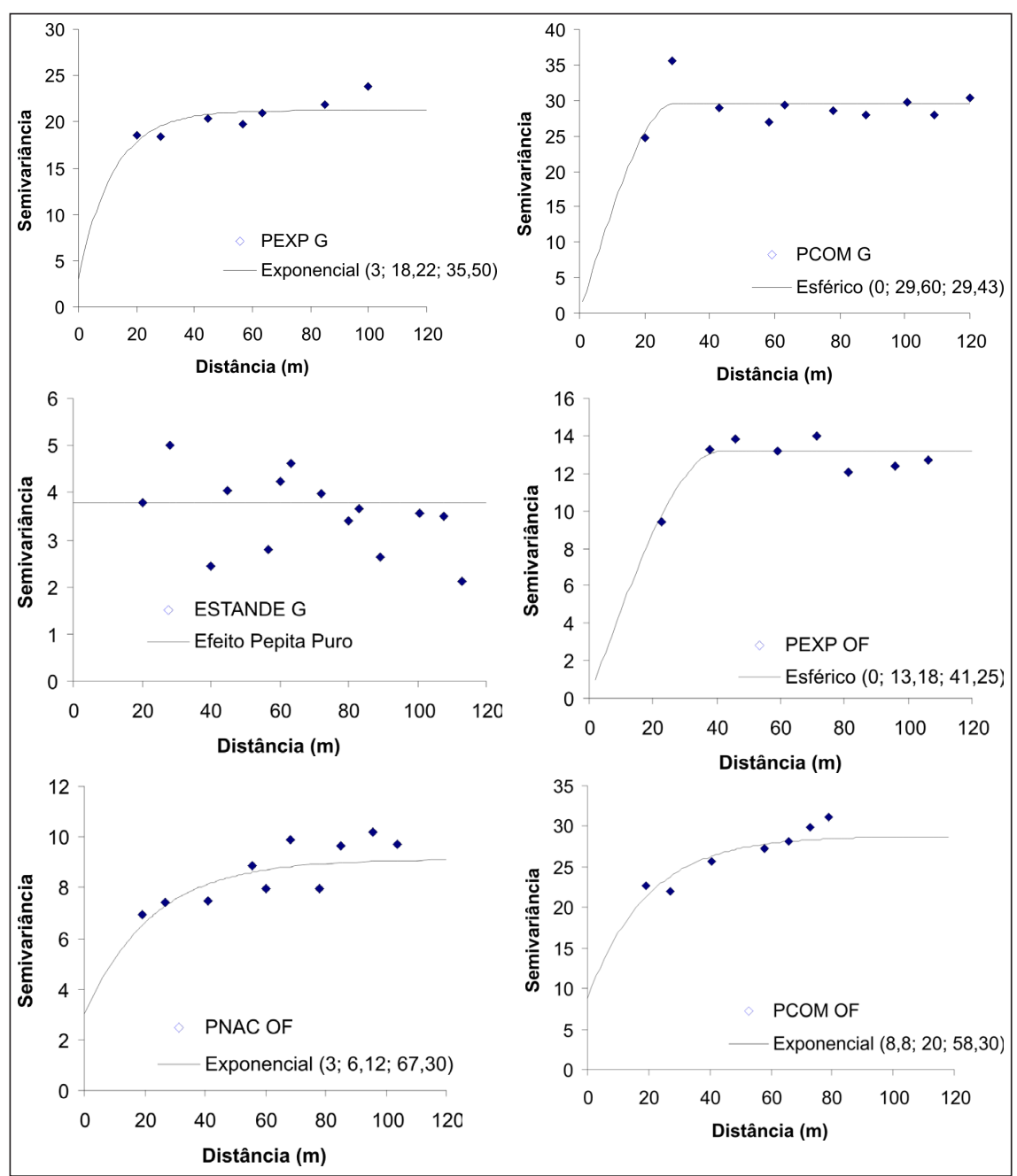

Figura 1. Semivariogramas omnidirecionais das produções de frutos de melão tipo exportação (PEXP G), de frutos comerciáveis (PCOM G) e estande do Goldex, e da produção de frutos tipo mercado nacional (PNAC OF), PEXP OF e PCOM OF do Orange Flesh. Mossoró, ESAM, 2002.

A PNAC foi superior à PEXP no 'Goldex', o que não é desejável, já que a produção de frutos tipo exportação dá maior retorno econômico. As produtividades obtidas nesse trabalho são consideradas baixas já que os produtores esperam PCOM maior do que $25 \mathrm{t} \mathrm{ha}^{-1}$, para ter lucro (PAIVA et al., 2003). Atualmente, alguns híbridos atingem produtividade de $41 \mathrm{t} \mathrm{ha}^{-1}$, como o melão amarelo Gold Mine em Carnaubais (RN) (ARAÚJO, 2000), e de $40 \mathrm{t} \mathrm{ha}^{-1}$ com o 'Orange Flesh' no Argissolo deste trabalho (BARROS, 2002).

As variáveis analisadas, com exceção do estande, apresentaram dependência espacial (Tabela 1). Para a confecção dos semivariogramas "omnidirecionais" foram eliminados, conforme Queiroz et al. (1999), alguns valores discrepantes de PNAC, nos dois casos, e de PEXP no 'Orange Flesh'. Isto praticamente não influenciou as medidas de posição e de dispersão, apenas a amplitude total.

$\mathrm{O}$ modelo efeito pepita puro obtido para estande (Figura 1) indica, segundo Trangmar et al. (1985), não haver correlação espacial entre amostras nas distâncias usadas. $\mathrm{O}$ modelo exponencial foi o que melhor se ajustou às variáveis PEXP e PNAC do 'Goldex' e PNAC e PCOM do 'Orange Flesh' (Figura 1), enquanto que o modelo esférico foi o que melhor se ajustou à PEXP no 'Orange Flesh' e PCOM no 'Goldex'.

A dependência espacial foi considerada forte, segundo critério de Cambardella et al. (1994), para PCOM do 'Goldex' e PEXP do 'Orange Flesh', que apresentaram uma relação efeito pepita/patamar (REPP) menor do que $1 \%$, e para PEXP no 'Orange Flesh' (16\%). As outras variáveis apresentaram dependência média. Segundo Vieira (2000) a baixa REPP de uma variável indica se tratar de fenômeno com grande continuidade espacial, o que resulta em menor variância da estimativa e maior confiança na interpolação.

Os alcances da dependência espacial de PNAC e PCOM tiveram os menores valores, ao redor de $30 \mathrm{~m}$ no Latossolo, e os maiores valores (67,3 m e 58,3 m) no Argissolo. Amostras localizadas a distâncias menores que o alcance são consideradas dependentes entre si, segundo Trangmar et al. (1985), e podem ser utilizadas na interpolação por krigagem.

A principal finalidade da modelagem da estrutura da dependência espacial através do semivariograma, segundo Queiroz et al. (1999), é possibilitar a estimativa por interpolação dos valores da variável para locais não amostrados e criar mapas de isovalores com maior precisão. As figuras com os isovalores do estande do 'Goldex' (Figura 2) 
apresentam regiões com menores valores na parte superior direita (X: 60-90 m e Y: 200-300 m) e maiores valores no centro da área, diminuindo para as laterais (X: 30-80 m e Y: 0-170 m; X: 0$60 \mathrm{~m}$ e Y: $230-300 \mathrm{~m})$. Apesar disso, a pequena amplitude destes dados não é suficiente para causar variação significativa em produtividade da cultura.

As regiões com menores PEXP do 'Goldex', as quais contribuem para a diminuição no volume total de frutos tipo exportação e na rentabilidade da área, estão na lateral esquerda e nos cantos superiores e inferiores da área. Os maiores valores de PEXP estão no centro da área, diminuindo para as laterais (X: 3080 m e Y: $80-170$ m; X: 40-70 m e Y: 230-300 m). Os frutos tipo exportação são prioridade na seleção, os que não se enquadram são comercializados no mercado nacional, obtendo menor remuneração, e os com sérios defeitos ou deterioração são refugados.

Os maiores valores de PNAC estão nas extremidades superior e inferior da área e coincidem com baixas PEXP. Como no 'Goldex' a PNAC tem maior média, são os valores de PEXP que estão muito baixos, e a localização destas regiões é importante para a identificação dos fatores prejudiciais. Os valores mais baixos de PNAC encontram-se em toda largura central da área, com ordenadas de 50 a $270 \mathrm{~m}$.

Existem regiões no centro (X: 4060 m e Y: 140-190 m) e na extremidade inferior da área do 'Goldex' (X: 30-70 $\mathrm{m}$ e Y: 0-80 m) onde altos valores de PCOM coincidem com altas PEXP e PNAC. As menores PCOM estão nas laterais (X: 0-30 m, 80-106 m e Y: 150270 m), que têm baixa PEXP e PNAC.

No 'Orange Flesh' (Figura 3), as regiões de menor estande estão na parte superior (X: 20-100 m e Y: 130-160 m) e central da área (X: 60-100 m e Y: 70$120 \mathrm{~m})$. Da mesma maneira que no 'Goldex', a amplitude é muito pequena para causar alguma influência sobre a produtividade.

Os menores valores de PEXP do 'Orange Flesh' se encontram em toda largura inferior, até a ordenada de $50 \mathrm{~m}$, e nos dois cantos superiores (X: 0-50 m,

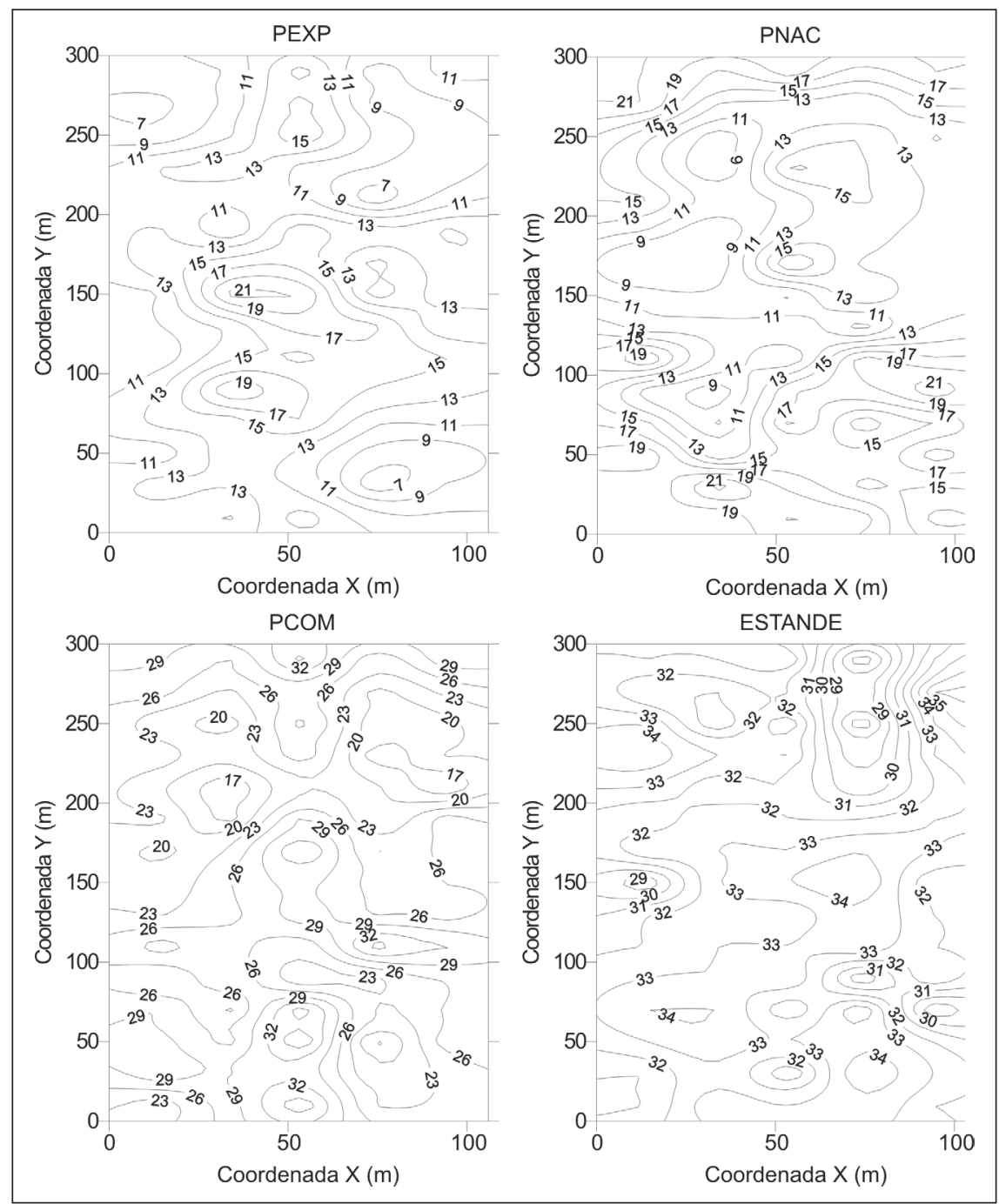

Figura 2. Isolinhas das produções em $\mathrm{t} \mathrm{ha}^{-1}$ de melão dos tipos exportação (PEXP), mercado nacional (PNAC) e comerciáveis (PCOM) e do número de plantas por parcela do meloeiro 'Goldex'. Mossoró, ESAM, 2002.

90-120 m e Y: 100-160 m), enquanto que os maiores valores estão no centro da área (X: 30-70 m e Y: 40-130 m). Nas duas áreas observa-se que os maiores valores do tipo exportação ocupam regiões centrais o que pode estar relacionado com a irrigação, já que são próximas aos pontos de alimentação.

As regiões com baixas PNAC do 'Orange Flesh' estão na parte inferior até a ordenada de $50 \mathrm{~m}$, continuando pela lateral esquerda até $100 \mathrm{~m}$. Os valores mais altos estão na parte superior, com exceção do canto esquerdo. Comparando com a figura de isolinhas de PEXP, as regiões com valores maiores de PNAC são poucas, o que seria desejável, porém a produção comerciável também é baixa nesta área.
A produção de frutos comerciáveis do 'Orange Flesh' apresentou valores baixos em toda largura inferior da área do até a ordenada de $50 \mathrm{~m}$, coincidindo com baixas PEXP e PNAC. A região superior central da área, com maior PCOM, coincide com altos valores de PEXP e PNAC. Apesar de serem híbridos diferentes, a menor média e a predominância de regiões com baixas PCOM no Argissolo indica existir influência mais intensa de fatores prejudiciais nesta área, que precisam ser identificados.

Foi constatada variabilidade espacial importante dos componentes de produção nas duas áreas, sugerindo sua correlação com fatores espacialmente distribuídos. A confecção de figuras com isovalores das variáveis de produção 


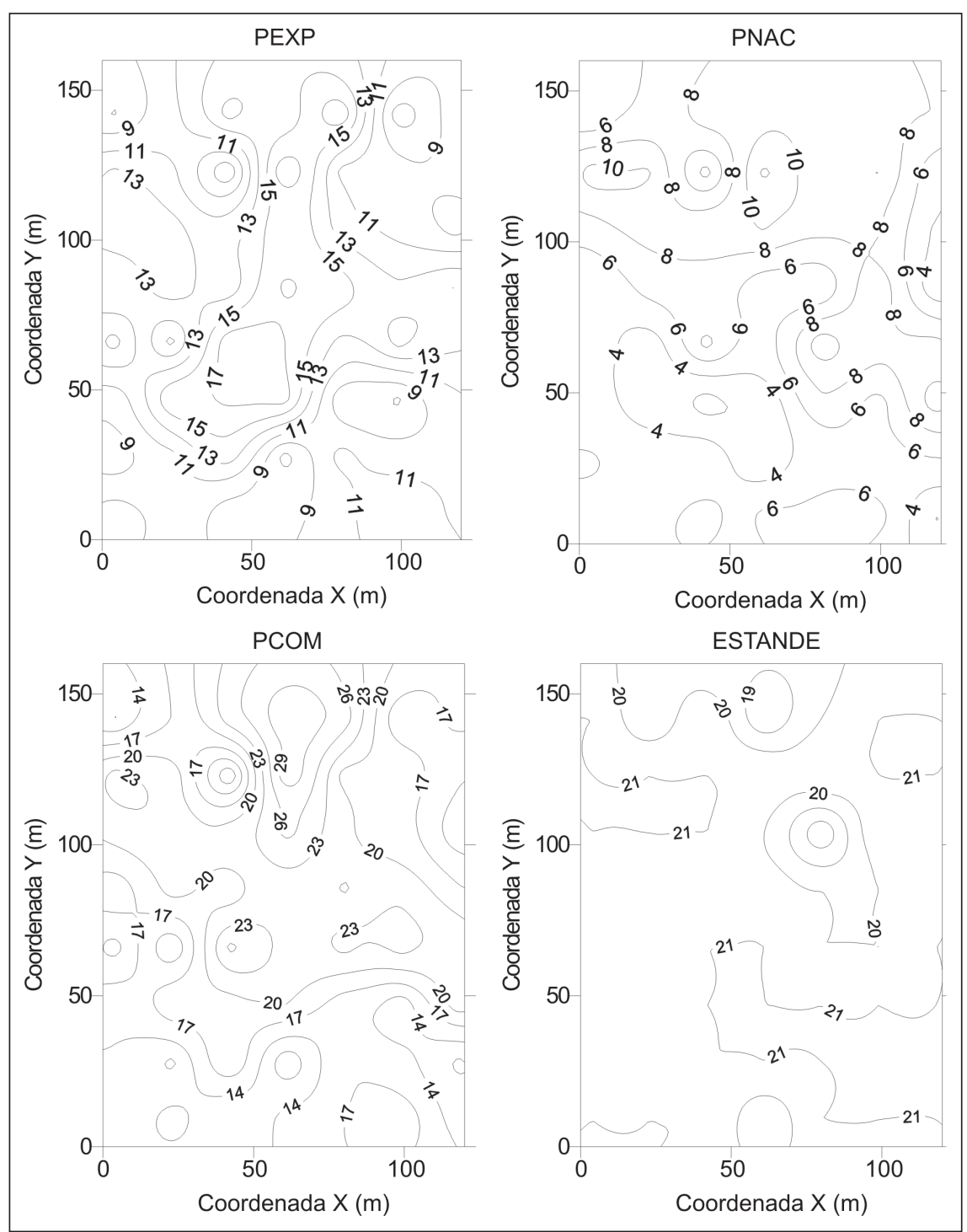

Figura 3. Isolinhas das produções em $\mathrm{t}_{\text {ha }}{ }^{-1}$ de melão dos tipos exportação (PEXP), mercado nacional (PNAC) e comerciáveis (PCOM) e do número de plantas por parcela do meloeiro 'Orange Flesh'. Mossoró, ESAM, 2002.

permitiu localizar as regiões do campo com baixo desempenho. Nestas regiões existe a possibilidade de aumento nas produções tipo exportação e comerciável e no retorno econômico da área como um todo, desde que sejam identificados os fatores limitantes e procedidas mudanças localizadas no manejo da cultura em aspectos como irrigação, fertilização, controle fitossanitário e descompactação.

\section{AGRADECIMENTOS}

À CAPES pelas bolsas concedidas, à FUNCAP pelo apoio financeiro e à Fazenda São João Ltda pelo uso de toda estrutura experimental.
CAMBARDELLA, C.A.; MOORMAN, T.B.; NOVAK, J.M.; PARKIN, T.B.; KARLEN, D.L.; TURCO, R.F.; KONOPKA, A.E. Field-scale variability of soil properties in central Iowa soils. Soil Science Society of America Journal, Madison, v.58, n.5, p.1501-1511, 1994.

CASSEL, D.K.; UPCHURCH, D.R.; ANDERSON, S.H. Using regionalized variables to estimate field variability of corn yield for four tillage regimes. Soil Science Society of America Journal, v.52, n.1, p.222-228, 1988.

CRISÓSTOMO, L.A.; SANTOS, A.A.; RAIJ, B.; FARIA, C.M.B.; SILVA, D.J.; FERNANDES, F.A.M.; SANTOS, F.J.S.; CRISÓSTOMO, J.R.; FREITAS, J.A.D.; HOLANDA, J.S.; CARDOSO, J.W.; COSTA, N.D. Adubação, irrigação, híbridos e práticas culturais para o meloeiro no Nordeste. Fortaleza: Embrapa Agroindústria Tropical, 2002. 21 p. Circular Técnica, 14.

ENGLUND, E.; SPARKS, A. Geo-EAS User's Guide. Las Vegas: U.S. Environmental Protection Agency, 1991. np. EPA/600/8-91/008.

JOHNSON, R.M.; BRADOW, J.M.; BAUER, P.J.; SADLER, E.J. Spatial variability of cotton fiber and quality in relation to soil variability. In: ROBERT, P.C.; RUST, R.H.; LARSON, W.E. Proceedings of the fourth international conference on precision agriculture. St. Paul: American Society of Agronomy, Crop Science Society of America, Soil Science Society of America, 1998. p.487-497.

MANTOVANI, E.C.; QUEIROZ., D.M.; DIAS, G.P. Máquinas e operações utilizadas na agricultura de precisão. In: SILVA, F.M.; BORGES, P.H.M. Mecanização e agricultura de precisão. Lavras: UFLA/SBEA, 1998. p.109-157. (Simpósio do XXVII Congresso Brasileiro de Engenharia Agrícola).

MARQUES JÚNIOR, J.; CORÁ, J.E. Atributos do solo para agricultura de precisão. In: SILVA, F.M.; BORGES, P.H.M. Mecanização e agricultura de precisão. Lavras: UFLA/SBEA, 1998. p.31-70. (Simpósio do XXVII Congresso Brasileiro de Engenharia Agrícola).

MORETTI, C.L.; ARAÚJO, J.L.P. Tecnologia de pós-colheita e comercialização. In: SILVA, H.R.; COSTA, N.D. Melão, produção aspectos técnicos. Brasília: Embrapa Hortaliças, Embrapa Semi-Árido, Embrapa Informação Tecnológica, 2003. p.121-129. MORTON, T.W.; BUCHLEITER, G.W.; HEERMANN, D.F. Quantifying the effects of water availability on corn yield under a centerpivot irrigation system. In: ROBERT, P.C.; RUST, R.H.; LARSON, W.E. Proceedings of the fourth international conference on precision agriculture. St. Paul: American Society of Agronomy, Crop Science Society of America, Soil Science Society of America, 1998. p.31-41.

NIELSEN, D.R.; KUTILEK, M.; WENDROTH, J.W.; HOPMANS, J.W. Selected research opportunities in soil physics. Scientia Agricola, Piracicaba, v.54, [número especial], p.51-77, 1997. PAIVA, W.O.; LIMA, J.A.A.; PINHEIRO NETO, L.G.; RAMOS, N.F.; VIEIRA, F.C. Melão Tupã: produtividade, qualidade do fruto e resistência a viroses. Horticultura Brasileira, Brasília, v.21, n.3, p-539-544, 2003.

PANNATIER, Y. Variowin: Software for spatial data analysis in 2D. New York: Springer-Verlag, 1996. 91 p. 
PLANT, R.E.; MERMER, A.; PETTYGROVE, G.S.; VAYSSIERES, M.P.; YOUNG, J.A.; MILLER, R.O.; JACKSON, L.F.; DENISON, R.F.; PHELPS, K. Factors underlying grains yield spatial variability in three irrigated wheat fields. Transactions of the ASAE, St. Joseph, v.42, n.5, p.1187-1202, 1999

QUEIROZ, J.E.; CRUCIANI, D.E.; LIBARDI, P.L. Variabilidade espacial da porosidade drenável de um solo de várzea, no município de Piracicaba, estado de São Paulo. Revista Brasileira de Engenharia Agrícola e Ambiental, Campina Grande, v.3, n.2, p.135-139, 1999.

RUNGE, E.C.A.; HONS, F.M. Precision agriculture - development of a hierarchy of variables influencing crop yields. In: ROBERT, P.C.; RUST, R.H.; LARSON, W.E. Proceedings of the fourth international conference on precision agriculture. St. Paul: American Society of Agronomy, Crop Science Society of America, Soil Science Society of America, 1998. p.143-158.
SCHUELLER, J.K.; WHITNEY, J.D. Precision agriculture for fruit trees. In: BORÉM, A.; GIÚDICE, M.P.; QUEIROZ, D.M.; MANTOVANI, E.C.; FERREIRA, L.R.; VALLE, F.X.R.; GOMIDE, R.L. Agricultura de precisão. Viçosa: UFV, 2000. p.321-338.

SEARCY, S.W. Agricultura de precisão: um desafio para a educação continuada. In: BALASTREIRE, L.A. O estado da arte da agricultura de precisão no Brasil. Piracicaba: L.A Balastreire, 2000. p.17-24.

SILVA, H.R.; COSTA, N.D. Introdução. In: SILVA, H.R.; COSTA, N.D. Melão, produção aspectos técnicos. Brasília: Embrapa Hortaliças, Embrapa Semi-Árido, Embrapa Informação Tecnológica, 2003. p.12-14.

STAFORD, J.V; LARK, R.M.; BOLAM, H.C Using yield maps to regionalize fields into potential management units. In: ROBERT, P.C.;
RUST, R.H.; LARSON, W.E. Proceedings of the fourth international conference on precision agriculture. St. Paul: American Society of Agronomy, Crop Science society of America, Soil Science Society of America. 1998. p. 225-237.

STOMBAUGH, T.S; SHEARER, S. Equipment technologies for precision agriculture. Journal of Soil and Water Conservation, v.55, n.1, p.6-11, 2000.

TRANGMAR, B.B.; YOST, R.S.; UEHARA, G. Application of geostatistics to spatial studies of soil properties. Advances in Agronomy, v.38, p.4594, 1985.

VIEIRA, S.R. Geoestatística em estudos de variabilidade espacial do solo. In: NOVAIS, R.F.; ALVAREZ, V.H.; SCHAEFER, C.E.G.R. Tópicos em ciência do solo. Viçosa: Sociedade Brasileira da Ciência do Solo, 2000. v.1, p.1-54.

WARRICK, A.W. Spatial variability. In: HILLEL, D. Environmental soil physics. San Diego: Academic Press, 1998. p.655-675. 